\title{
When good advertisement backfires: cleaners get eaten too
}
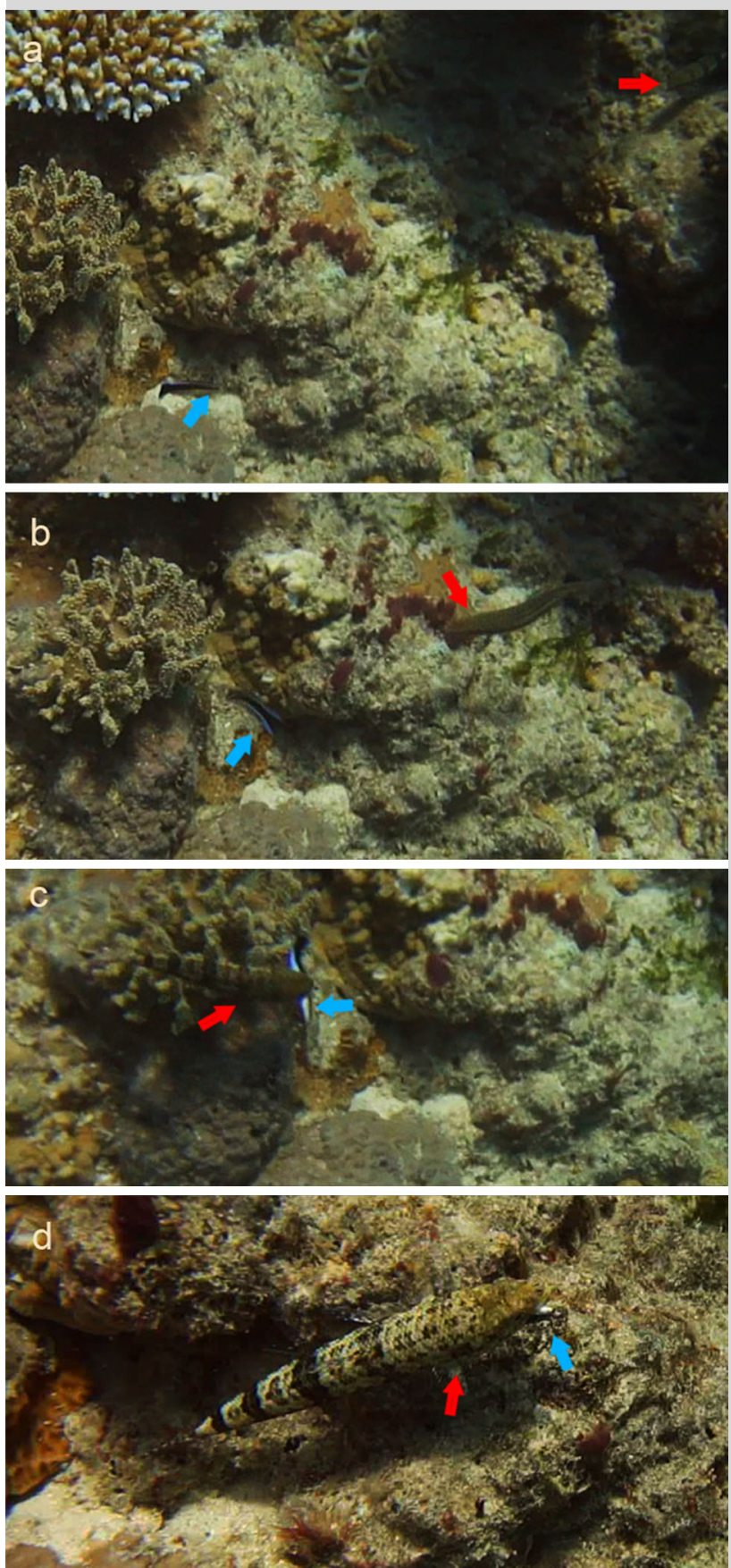

Fig. 1 Series of four photographs (see movie in Electronic Supplementary Materials) of a lizardfish, Synodus sp., preying on a cleaner wrasse, Labroides dimidiatus. Red arrows indicate the lizardfish location, and blue arrows the cleaner wrasse's. Lizardfish is spotted waiting (a); it then lunges to the cleaner (b), catches it (c), and finally starts swallowing it (d)
Cleaning interactions found in coral reef communities are a textbook example of mutualistic behavior; cleaners provide a service to larger, so-called client fish by removing and ingesting ectoparasites. Specific coloration is important to signal the occurrence of these interactions and indeed, all cleaner species share the particular feature of sporting colorful, contrasting stripes which they use to advertise their cleaning services to clients, combined with tactile dancing (Grutter 2004; Cheney et al. 2009; Lettieri and Streelman 2010). Among cleaner fish species, blue stripes that provide the highest contrast in coral reef environments and lead to better visibility for potential partners (Cheney et al. 2009) are solely found in obligate cleaners (Lettieri and Streelman 2010). However, successful interactions depend greatly on the potential clients' intentions, as this increase in exposure comes with the added risk of predation. Although predatory clientele react more to high-contrast blue stripes (Lettieri and Streelman 2010), it is thought that cleaners are almost immune to predation due to the ability to manipulate their clients (Grutter 2004), in addition to specific coloration that may provide extra protection (Cheney et al. 2009; Lettieri and Streelman 2010). Here, we show first-hand footage of an Indo-Pacific bluestreak cleaner wrasse, Labroides dimidiatus, falling prey to a lizardfish, Synodus sp. (photo series in Fig. 1; see Electronic Supplementary Material for full video), thus providing evidence that cleaner fish do suffer risk and are eaten at times. This predatory event was observed in 2012 on Mermaid Reef, Lizard Island (Lizard Island Research Station, Australia, $14^{\circ} 40^{\prime} \mathrm{S}, 145^{\circ} 28^{\prime} \mathrm{E}$ ), within the cleaner wrasse's territory (cleaning station), but not during or following a cleaning event.

\section{References}

Cheney KL, Grutter AS, Blomberg SP, Marshall NJ (2009) Blue and yellow signal cleaning behavior in coral reef fishes. Curr Biol 19:1283-1287

Grutter AS (2004) Cleaner fish use tactile dancing behavior as a preconflict management strategy. Curr Biol 14:1080-1083

Lettieri L, Streelman JT (2010) Colourful stripes send mixed messages to safe and risky partners in a diffuse cleaning mutualism. J Evol Biol 23:22892299

Electronic supplementary material The online version of this article (doi:10.1007/s00338-015-1303-1) contains supplementary material, which is available to authorized users.

J. P. M. Messias $(\bowtie)$ · M. C. Soares

Centro de Investigação em Biodiversidade e Recursos Genéticos

(CIBIO), Universidade do Porto, Campus Agrário de Vairão, 4485-661

Vairão, Portugal

e-mail: joao.messias@ cibio.up.pt 